\title{
BMJ Open Design and reporting characteristics of clinical trials investigating sedation practices in the paediatric intensive care unit: a scoping review by SCEPTER (Sedation Consortium on Endpoints and Procedures for Treatment, Education and Research)
}

\author{
Jennifer Jooyoung Lee (10 , ${ }^{1}$ Jerri C Price, ${ }^{1}$ Jennifer Gewandter, ${ }^{2}$ \\ Bethea A Kleykamp, ${ }^{2}$ Katherine V Biagas, ${ }^{3}$ Maryam Y Naim (D) , ${ }^{4}$ Denham Ward, ${ }^{2}$ \\ Robert H Dworkin, ${ }^{2}$ Lena S Sun ${ }^{5}$
}

To cite: Lee JJ, Price JC, Gewandter J, et al. Design and reporting characteristics of clinical trials investigating sedation practices in the paediatric intensive care unit: a scoping review by SCEPTER (Sedation Consortium on Endpoints and Procedures for Treatment, Education and Research). BMJ Open 2021;11:e053519. doi:10.1136/ bmjopen-2021-053519

- Prepublication history and additional supplemental material for this paper are available online. To view these files, please visit the journal online (http://dx.doi.org/10.1136/ bmjopen-2021-053519).

Received 14 May 2021

Accepted 24 September 2021

Check for updates

(c) Author(s) (or their employer(s)) 2021. Re-use permitted under CC BY-NC. No commercial re-use. See rights and permissions. Published by BMJ.

For numbered affiliations see end of article.

Correspondence to

Dr Lena S Sun;

Iss4@cumc.columbia.edu

\section{ABSTRACT}

Objectives To conduct a scoping review of sedation clinical trials in the paediatric intensive care setting and summarise key methodological elements.

Design Scoping review.

Data sources PubMed, Embase, Cumulative Index to Nursing and Allied Health Literature and grey references including ClinicalTrials.gov from database inception to 3 August 2021.

Study selection All human trials in the English language related to sedation in paediatric critically ill patients were included. After title and abstract screening, full-text review was performed. 29 trials were eligible for final analysis. Data extraction A coding manual was developed and pretested. Trial characteristics were double extracted. Results The majority of trials were single centre (22/29, $75.9 \%)$, parallel group superiority $(17 / 29,58.6 \%)$, doubleblinded $(18 / 29,62.1 \%)$ and conducted in an academic setting $(29 / 29,100.0 \%)$. Trial enrolment $(\geq 90 \%$ planned sample size) was achieved in $65.5 \%$ of trials (19/29), and retention ( $\geq 90 \%$ enrolled subjects) in $72.4 \%$ of trials $(21 / 29)$. Protocol violations were reported in nine trials $(31.0 \%)$. The most commonly studied cohorts were mechanically ventilated patients $(28 / 29,96.6 \%)$ and postsurgical patients $(11 / 29,37.9 \%)$ with inclusion criteria for age ranging from $0 \pm 0.5$ to $15.0 \pm 7.3$ years (median $\pm I Q R$ ). The median age of enrolled patients was 1.7 years (IQR=4.4 years). Patients excluded from trials were those with neurological impairment (21/29, $72.4 \%)$, complex disease $(20 / 29,69.0 \%)$ or receipt of neuromuscular blockade (10/29, 34.5\%). Trials evaluated drugs/protocols for sedation management $(20 / 29,69.0 \%)$, weaning $(3 / 29,10.3 \%)$, daily interruption $(3 / 29,10.3 \%)$ or protocolisation $(3 / 29,10.3 \%)$. Primary outcome measures were heterogeneous, as were assessment instruments and follow-up durations.

Conclusions There is substantial heterogeneity in methodological approach in clinical trials evaluating

\section{Strengths and limitations of this study}

- This is the first scoping review of the literature that has identified all sedation-related clinical trials in the critically ill paediatric population and summarised key methodological elements.

- An extensive, up-to-date search strategy using three databases and grey references was conducted followed by study selection and double data extraction to evaluate inclusion and exclusion criteria, intervention characteristics, measurement instruments and efficacy outcome measures.

- This review was limited by the exclusion of publications in languages other than English, in addition to prospective observational and other interventional studies such as before-and-after studies given the aim of the current study was to assess clinical trial design and reporting.

- Considerations for future trial design and conduct are proposed based on this review's findings, but these may need to be adapted to be applied to the unique aspects of individual trial objectives.

- Input and consensus from various stakeholders is needed to establish a core set of sedation trial design recommendations and outcome domains in paediatric critical care patients, which may improve the quality and comparability of future trials and aid in the development of sedation-related clinical guidelines.

sedation in critically ill paediatric patients. These results provide a basis for the design of future clinical trials to improve the quality of trial data and aid in the development of sedation-related clinical guidelines. 


\section{INTRODUCTION}

Sedation of critically ill paediatric patients in the intensive care unit provides anxiolysis and mitigates the stress response, in addition to facilitating the tolerance of respiratory support, invasive procedures and nursing care. ${ }^{1}$ An optimal sedation approach can be described as one with ease of initiation and titration, high efficacy and costeffectiveness, while allowing rapid recovery after discontinuation with minimal adverse effects. ${ }^{2}$

Consensus guidelines, at present, acknowledge the lack of high-quality evidence on which to base practice recommendations for sedation. ${ }^{3-8}$ This lack of evidence has resulted in heterogeneous approaches to management $^{9-11}$ and widespread off-label sedative use across paediatric intensive care units (PICUs) ${ }^{12-15}$ For example, a recent systematic review ${ }^{16}$ found that optimal sedation was only achieved in little over half $(57.6 \%)$ of the time. Of those with suboptimal sedation, assessments found oversedation $(31.8 \%)$ occurring more frequently than undersedation $(10.6 \%)$.

Although robust evidence from clinical trials is important in establishing consensus guidelines for optimal sedation in critically ill children, trials remain scarce due to numerous barriers that enhance the complexity of clinical research in this practice area. Tremendous variation in clinical practice exists across medical centres, thereby making the determination of equipoise extremely challenging. In addition, the constraints of clinical care inherent to the high acuity PICU environment lead to challenges in patient recruitment, retention and implementation of intervention in clinical trials.

Existing paediatric critical care trials have been reported to vary widely in their design, conduct and reporting practices. ${ }^{17}$ This in turn impedes the ability to meaningfully synthesise results across studies and generate practice recommendations. Recent reviews and recommendations by the Sedation Consortium on Endpoints and Procedures for Treatment, Education and Research are the first to attempt to provide a foundation to guide the design and evaluation of adult and paediatric sedation clinical trials. ${ }^{18-22}$

In this scoping review, we identified all sedation-related clinical trials in the critically ill paediatric population to assess the current state of key methodological elements. This review focuses on summarising inclusion and exclusion criteria, intervention characteristics, measurement instruments and efficacy outcome measures. The aims of this review are twofold: (1) to identify existing knowledge gaps and challenges related to clinical trial design, particularly those applicable to the paediatric population and (2) to discuss the implications of our findings for future research design. The results of this review have the potential to improve the quality of design and reporting of future sedation-related clinical trials, and promote the interpretability and synthesis of results to aid in the establishment of evidence-based practice guidelines.

\section{METHODS}

\section{Study selection}

The Preferred Reporting Items for Systematic Reviews and Meta-Analyses Extension (PRISMA) for Scoping Reviews Checklist was used. ${ }^{23}$ This scoping review was not preregistered; it was registered post hoc on Open Science Framework (available at: https://osf.io/mnezu). PubMed, Embase and the Cumulative Index to Nursing and Allied Health Literature (CINAHL) were searched from database inception through 3 August 2021 for all human clinical trials related to sedation in the PICU setting. Truncated search terms with variations in spelling were used for 'paediatric critical care,' 'sedatives' and 'analgesics' for PubMed with a filter for human clinical trials. These were then translated to the corresponding languages to search Embase and CINAHL (detailed search strategies in online supplemental appendix 1). Clinical trials studying the practice of sedation and/or analgesia, where it was deemed to be a subsidiary component to sedation (eg, continuous opioid infusions administered to intubated, mechanically ventilated patients), were considered to be eligible. Trials published in the English language with full text availability were included. A reference librarian was consulted to review our search strategies, which were optimised through an iterative process of examining the literature and testing the search strategy to ensure maximal capture of pertinent studies.

Sedation-related studies specifically examining assessment scales, pharmacokinetics or pharmacodynamics, or non-pharmacological interventions were not included. We also excluded studies that investigated anaesthetic, sedative or analgesic practices in the operating arena or for intubation and other specific procedures only. Observational studies were omitted, as were trials solely including adults (age greater than 21 years), premature infants or infants in the neonatal ICU. Trials that did not have a control or active intervention (also referred to as the 'comparator') arm, in addition to before-and-after studies were also excluded.

Search hits were screened by title and abstract then independently examined through full text review by two authors (JJL or JCP) for inclusion using the Covidence software. ${ }^{24}$ A snowballing strategy of inspecting reference lists of key articles was also used to identify relevant studies that may have been missed. An earlier scoping review $^{25}$ that identified all published trials in paediatric critical care was examined, and five additional references were added to be screened. ClinicalTrials.gov was also searched; eight unique trials that were not matched to existing publications were identified. These trials are summarised separately, but were not included in the final analysis as there were insufficient data available to extract for the purposes of this scoping review (online supplemental table 1).

\section{Data extraction}

A coding manual was developed to extract general trial characteristics, eligibility criteria, intervention 
characteristics and efficacy outcome measures (online supplemental appendix 2). Information on safety and adverse events reporting was not included because these outcomes were beyond the scope of this review. The coding manual was pretested and modified for content and structure in multiple rounds using trials that met inclusion criteria prior to formal extraction. Information was then coded from articles by two authors (JJL or JCP) for final analysis. Discrepancies in coding were discussed among both authors and adjudicated by discussion or a third author (LSS) when necessary.

\section{Statistical analyses}

All statistical analyses were performed in Microsoft Excel V.15.23. Descriptive statistics were used to summarise trial design and reporting features. Continuous data are reported as median and IQR (IQR=third quartile-first quartile), or range (minimum, maximum). Categorical data are reported as count and percentage (\%) where the number of total trials (by subcategory where applicable) was used in the denominator. Some trials had elements which fell into multiple categories, which account for instances in which the count exceeds the total number of trials.

\section{Patient and public involvement}

Patients or the public were not involved in the design, or conduct, or reporting, or dissemination plans of our research.

\section{RESULTS}

In total, there were 1676 unique search hits. After initial title and abstract screening, 165 studies were assessed for full-text eligibility. One hundred and four studies were excluded on the basis of study design (78 observational studies, 20 before-and-after studies, 6 reviews). Other reasons for exclusion included full-text unavailability, non-English language, absent or excluded intervention and comparator (eg, studies solely investigating assessment scales, pharmacokinetics or pharmacodynamics, non-pharmacologic interventions, anaesthetic, sedative or analgesic practices in the operating arena or for intubation and other specific procedures), or wrong patient population (eg, neonates or adults only). Ultimately, 29 trials were included in this scoping review for final analysis. ${ }^{26-54}$ The PRISMA flow diagram is depicted in figure 1.

Figure 1 PRISMA flow diagram. Study selection details are presented. Twenty-nine trials were eligible for final analysis. PRISMA, Preferred Reporting Items for Systematic Reviews and Meta-Analyses. 


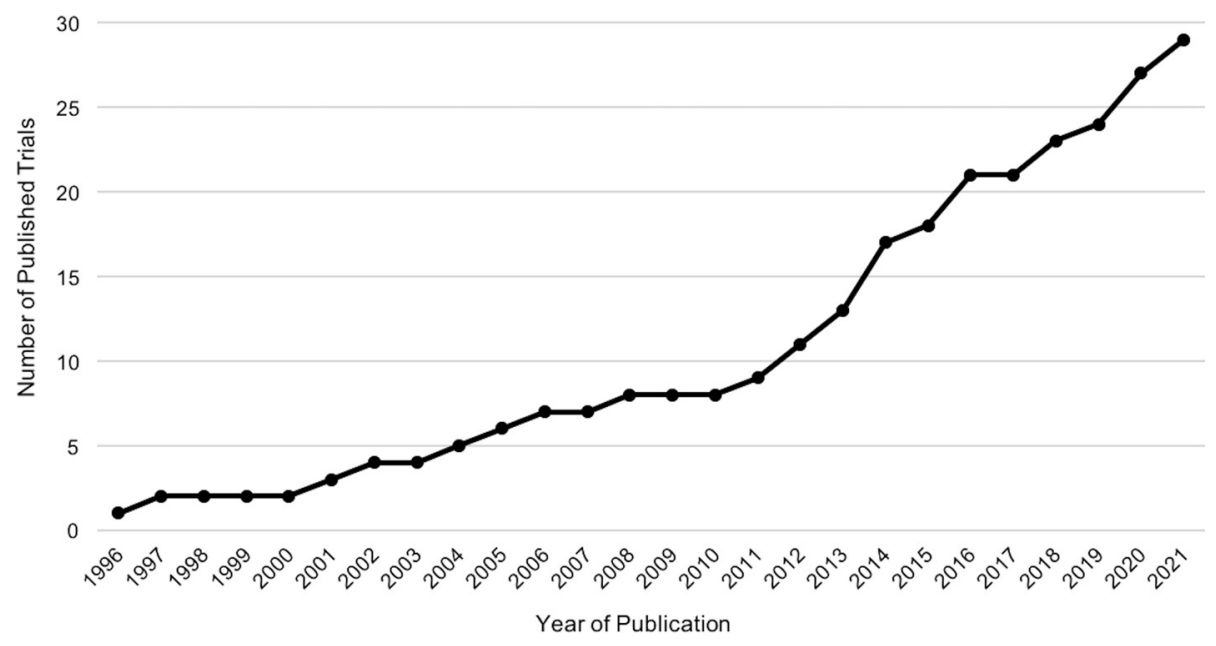

Figure 2 Paediatric sedation trial publications. This figure shows the rising trend in number of paediatric sedation trial publications per year from 1996 to 2021.

\section{Trial characteristics}

The majority of trials were single centre $(22 / 29,75.9 \%)$, double-blinded $(18 / 29,62.1 \%)$ and a parallel group superiority design $(17 / 29,58.6 \%)$. Eight trials $(27.6 \%)$ were not blinded in any fashion, and two trials $(6.9 \%)$ were single-blinded only for outcome assessor. In four trials $(13.8 \%)$, the design could not be determined, and there was no sample size or power calculation reported.

All trials were conducted in an academic or tertiary care hospital setting, spanning various continents (North America 8/29, 27.6\%; South America 1/29, 3.4\%; Europe 9/29, 31.0\%; Asia 8/29, 27.6\%; Africa 2/29, 6.9\%; other $1 / 29,3.4 \%)$. Sponsorship was reported in all but six trials $(20.7 \%)$. Trials were sponsored by government agencies $(7 / 29,24.1 \%)$, professional organisations/foundations $(8 / 29,27.6 \%)$, institutions or universities $(3 / 29,10.3 \%)$, or industry $(3 / 29,10.3 \%)$.

The vast majority of trials $(21 / 29,72.4 \%)$ were published after 2009 (figure 2). Trial duration lasted at least 1 year in 18 trials $(62.1 \%)$. In the trials that reported sample size calculation $(25 / 29,86.2 \%)$, the median planned sample size was $\mathrm{n}=60$ with an IQR of $93.34 .5 \%(10 / 29)$ of trials were not able to achieve $\geq 90 \%$ planned enrolment and may, therefore, have been underpowered. $72.4 \%$ (21/29) of trials reached $\geq 90 \%$ completion rate. Trial flow details were not consistently available, as $67.9 \%(n=19)$ of the 28 trials, which were published after the release of the Consolidated Standards of Reporting Trials (CONSORT) statement, ${ }^{55}$ provided a trial flow diagram.

In terms of other specific design characteristics, fewer than one-third of trials reported any use of pretrial staff training $(6 / 29,20.7 \%)$, a run-in period, which was defined as a test period in which the intervention was implemented prior to formal outcome assessment $(4 / 29$, $13.8 \%)$, or assessment of adherence by staff $(9 / 29,31.0 \%)$. Early termination occurred in three trials $(10.3 \%)$ and was attributable to an adverse event $(1 / 3,33.3 \%)$, futility $(1 / 3,33.3 \%)$ or slow recruitment $(1 / 3,33.3 \%)$. General trial characteristics are summarised in table 1.

\section{Eligibility criteria}

While the inclusion criteria for age was wide (range $0 \pm 0.5$ to $15.0 \pm 7.3$ years; not reported $2 / 29,6.9 \%$ ), the ages of enrolled patients for the trials were young (1.7 \pm 4.4 years; not reported $2 / 29,6.9 \%)$. Almost all of the trials $(28 / 29,96.6 \%)$ studied intubated, mechanically ventilated patients. The single trial that did not identify mechanical ventilation as a criterion for inclusion was a weaning trial. ${ }^{27}$ Of the 28 studies of mechanically ventilated patients, 6 trials $(21.4 \%)$ examined patients with anticipated early extubation (ie, within 24-48hours), and 11 studies $(39.3 \%)$ examined those who needed prolonged intubation (ie, greater than $24-48$ hours). In the remaining 11 trials, the duration of mechanical ventilation was not specified. In terms of clinical patient type, post-surgical cardiac $(5 / 29,17.2 \%)$ and posturgical noncardiac $(6 / 29,20.7 \%)$ patients were the most commonly studied. A mixed medical and postsurgical population was examined in five trials $(17.2 \%)$.

Table 2 lists all trial inclusion and exclusion criteria extracted in detail. The most common exclusion criteria that trials reported were: inability to evaluate level of sedation (eg, neurological disease) (21/29, 72.4\%), history of drug dependence or withdrawal and previous receipt of sedatives $(13 / 29,44.8 \%)$, receipt of neuromuscular blockers $(10 / 29,34.5 \%)$ or known allergy or adverse reaction to trial drugs $(10 / 29,34.5 \%)$. Severely ill, complex patients (eg, those with haemodynamic instability, major end-organ dysfunction, limited life expectancy, status postemergency or complex surgeries and reoperations, or clinical indications which preclude patient arousal such as open chest, pulmonary hypertension, difficult airway) were also frequently excluded $(20 / 29,69.0 \%)$. Discussion of generalisability occurred in approximately half of the trials $(17 / 29,58.6 \%)$. The effectiveness of randomisation was evaluated in nearly all trials $(28 / 29,96.6 \%)$ with $72.4 \%$ (21/29) demonstrating a balanced distribution of characteristics between control and comparator groups. 
Table 1 Trial characteristics

Publication year (n, \%)

1996-2008

2009-2021

$21(72.4)$

Trial duration ( $\mathrm{n}, \%)$

Less than 1 year

2 (6.9)

1-2 years

9 (31.0)

Greater than 2 years

9 (31.0)

Not reported

9 (31.0)

Trial location (n, \%)

\begin{tabular}{|lc|}
\hline North America & $8(27.6)$ \\
\hline South America & $1(3.4)$ \\
\hline Europe & $9(31.0)$ \\
\hline Asia & $8(27.6)$ \\
\hline Africa & $2(6.9)$ \\
\hline Other & $1(3.4)$ \\
\hline Trial design (n, \%) & \\
\hline Parallel group superiority & $17(58.6)$ \\
\hline Parallel group non-inferiority & $1(3.4)$ \\
\hline Cluster randomised & $2(6.9)$ \\
\hline Pilot trial & $4(13.8)$ \\
\hline Equivalence & $1(3.4)$ \\
\hline Unclear & $4(13.8)$ \\
\hline
\end{tabular}

Site characteristics (n, \%)

$\begin{array}{lr}\text { Single centre } & 22(75.9) \\ 2-5 \text { centres } & 4(13.8) \\ >5 \text { centres } & 3(10.3)\end{array}$

Clinical setting ( $\mathrm{n}, \%)$

Academic or tertiary care hospital

29 (100.0)

Sponsort (n, \%)

Industry

Institution or university

$3(10.3)$

Government agency

$7(24.1)$

Professional organisation or foundation

8 (27.6)

None

$6(20.7)$

Not reported

$6(20.7)$

Blinding ( $\mathrm{n}, \%)$

Single (only outcome assessor was blinded)

$2(6.9)$

Double-blind (patient, intervention $18(62.1)$

administrator and outcome assessor were blinded)

None

8 (27.6)

Not reported

$1(3.4)$

No of trials that reported sample size calculation (n, \%)

Planned sample size (median, IQR)

No of patients enrolled (median, IQR)
$60(93)$

25 (86.2)

$60(68)$

Continued
Table 1 Continued

No of trials that enrolled $\geq 90 \%$ of planned

$19(65.5)$

sample size (n, \%)

No of patients that completed the trial (median, 57 (75)

IQR)

No of trials in which $\geq 90 \%$ of enrolled patients 21 (72.4) completed the trial $(n, \%)$

No of trials that provided the CONSORT flow 19 (67.9) diagram $\neq(n, \%)$

No of trials that implemented pretrial training $\quad 6(20.7)$ $(n, \%)$

No of trials with a run-in period (n, \%)

$4(13.8)$

No of trials that assessed adherence $(n, \%)$

$9(31.0)$

Trial stopped early (n, \%)

$3(10.3)$

Adverse event

Futility

Slow recruitment

$1(33.3)$

General trial design characteristics are summarised.

*Pilot trials were identified as such by the authors in the publication and designed primarily to evaluate for safety and feasibility. †Trials had multiple types of sponsors, which account for total number of trials exceeding $n=29$.

$\ddagger$ Twenty-eight trials were published after the initial CONSORT statement was available in 1996, therefore, $n=28$ was used as the denominator to calculate the percentage.

CONSORT, Consolidated Standards of Reporting Trials.

\section{Intervention characteristics}

Trial interventions included comparison of drugs and/ or protocols for sedation management $(20 / 29,69.0 \%)$, sedation weaning $(3 / 29,10.3 \%)$, daily interruption of sedation $(3 / 29,10.3 \%)$ and the implementation of a protocolised sedation regimen $(3 / 29,10.3 \%)$. Detailed information on intervention characteristics is presented in table 3 .

Providers who administered the intervention were a combination of physicians and nurses in 10 trials $(34.5 \%)$, physicians, nurses and ancillary staff in 3 trials (10.3\%), physicians alone in 8 trials $(27.6 \%)$, nurses alone in 5 trials $(17.2 \%)$ and not reported in 3 trials $(10.3 \%)$. Among the 28 trials examining sedation in intubated, mechanically ventilated patients, 10 trials $(35.7 \%)$ described ventilator management strategies in their methodology. In all 29 trials included in the review, control and comparator groups received the same types of secondary sedatives in 17 studies $(58.6 \%)$ and different types of agents in 7 studies (24.1\%). Neuromuscular blockade was mostly either not administered $(11 / 29,37.9 \%)$, as prespecified in the trial exclusion criteria, or was not detailed (14/29, $48.3 \%$ ). Duration of follow-up was extremely heterogeneous, but mostly short term, on the order of days (table 3).

\section{Efficacy assessment and outcome measures}

Sedation was assessed using widely variable instruments, including the COMFORT $(7 / 29,24.1 \%)$ or 
Table 2 Patienteligibility criteria

Age range for inclusion criteria (years) (median, Min 0 IQR)

(0.5), max

$15.0(7.3)$

Not reported (n, \%)

2 (6.9)

Age of enrolled patients (years) (median, IQR) $1.7(4.4)$

Not reported $(\mathrm{n}, \%)$

$2(6.9)$

Trial inclusion criteria* $(n, \%)$

Intubated, mechanically ventilated

28 (96.6)

Early extubation (24-48 hours)

6 (21.4)

Prolonged intubation (>24-48 hours)

11 (39.3)

Timeframe of extubation not specified

$11(39.3)$

Patient type

Postsurgical cardiac patients only

$5(17.2)$

Postsurgical non-cardiac patients only

$6(20.7)$

Medical patients only

2 (6.9)

Mixed medical and post-surgical patients,

2 (6.9)

both non-cardiac

Mixed medical and postsurgical patients, both $3(10.3)$ cardiac and non-cardiac

Trauma patients

$2(6.9)$

Trial exclusion criteria* $(n, \%)$

Inability to evaluate level of sedation (eg,

$21(72.4)$

neurological disease)

Use of neuromuscular blockers

$10(34.5)$

Contraindications to arousal (eg, pulmonary

hypertension, difficult)

7 (24.1)

Airway, open chest)

Limited life expectancy (eg, do-not-resuscitate 5 (17.2) order)

Status-postcardiac arrest

2 (6.9)

Consideration for organ procurement

$1(3.4)$

Haemodynamic instability (eg, use of

$7(24.1)$

vasopressors, inotropes)

Respiratory complications

3 (10.3)

Other major end-organ dysfunction

$19(65.5)$

Chronic hypertension

$3(10.3)$

History of drug dependence or withdrawal, $\quad 13$ (44.8) previous receipt of sedatives

$\begin{array}{ll}\text { Pregnancy } & 4 \text { (13.8) } \\ \text { Enrolment in another conflicting trial or } & 5(17.2)\end{array}$
previous enrollment in current trial

Known allergy or adverse reaction to trial 10 (34.5) drug(s)

Prematurity

$1(3.4)$

Emergency or complex surgeries, reoperation 5 (17.2)

No of trials that discussed generalisability (n, \%) 17 (58.6)

Severity of illness evaluated* $(n, \%)$

Yes, by use of score (eg, Paediatric Risk of

$23(79.3)$

Mortality, Paediatric Index of Mortality
Table 2 Continued

Paediatric Logistic Organ Dysfunction)

Yes, by assessing comorbid conditions

$24(82.8)$

No

$1(3.4)$

Presence of significant baseline differences in

control and comparator groups (n, \%)

$\begin{array}{lc}\text { Yes } & 7(24.1) \\ \begin{array}{l}\text { Differences accounted for (eg, subgroup } \\ \text { analysis) }\end{array} & 2(28.6) \\ \text { No } & 21(72.4) \\ \text { Not reported } & 1(3.4)\end{array}$

Trial inclusion/exclusion criteria by patient demographic and clinical characteristics are outlined.

*Trials had multiple inclusion/exclusion criteria and ways of evaluating severity of illness, which account for total number of trials exceeding $n=29$. Extracted trial inclusion/exclusion criteria include most common factors cited in the literature and were prespecified in the coding manual.

COMFORT-Behaviour Scale (6/29, 20.7\%), Ramsay/ Modified Ramsey Sedation Scale $(7 / 29,24.1 \%)$, State Behaviour Scale $(4 / 29,13.8 \%)$, PICU Sedation Scale $(4 / 29,13.8 \%)$ and Tracheal Suctioning Score $(4 / 29$, $13.8 \%$ ) (table 4). The COMFORT $(5 / 14,35.7 \%)$ or COMFORT-Behaviour Scale $(5 / 14,35.7 \%)$ was most frequently used in trials in infants $(\mathrm{n}=14)$, in which the median age of enrolled patients was under 2 years, compared with trials in children or adolescents $(n=13)$, in which the median age of enrolled patients was $5.0 \pm 6.2$ years. ${ }^{56}$ In the 13 trials in children and adolescents, the Ramsay/Modified Ramsay Sedation Scale was most commonly utilised $(6 / 13,46.2 \%)$.

Differences in the use of assessment tools were found based on the geographic location where the trials were conducted. Trials in Europe $(\mathrm{n}=9)$ used the COMFORT Scale in five trials $(55.6 \%)$ and COMFORT-Behaviour Scale in four trials (44.4\%). The Ramsay/Modified Ramsay Sedation Scale was most frequently employed to assess sedation in other continents (North America 2/7, 28.6\%; Asia 3/8, 37.5\%; Africa 2/2, 100.0\%). Detailed age and geographic distributions of sedation assessment methods are presented in online supplemental table 2. Pain assessment method, distinct from that of sedation, was not described in $62.1 \%$ of trials $(18 / 29)$. The Faces, Legs, Activity, Cry, Consolability (or Modified) Score was used when pain was specifically assessed separately from sedation in six trials $(20.7 \%)$.

There was no first party or patient reporting of sedation and/or pain levels. Second party reporting, of which most commonly involved nurse assessment $(18 / 29,62.1 \%)$, was typically performed in regular frequent intervals (every $2-4$ hours 19/29, 65.5\%; every 1 hour or more often $7 / 29$, $24.1 \%$ ). Sedation assessment and management in both control (25/29, 86.2\%; 22/29, $75.9 \%$, respectively) and 
Table 3 Intervention characteristics

Type of intervention (n, \%)

Drug/protocol for sedation

$20(69.0)$

Remifentanil versus fentanyl

$1(5.0)$

Naloxone+fentanylversus fentanyl

$1(5.0)$

Midazolam+fentanyl (combined vs separate $\quad 1$ (5.0) solutions)

Midazolam+fentanylversus midazolam

$1(5.0)$

Midazolam (high vs low dose)

$1(5.0)$

Chloral hydrate+promethazine versus

$1(5.0)$

midazolam

Morphine+midazolam

$1(5.0)$

(continuous+intermittent vs intermittent only)

Morphine (intermittent vs continuous) 2 (10.0)

Dexmedetomidine versus midazolam 3 (15.0)

Dexmedetomidine versus fentanyl 3 (15.0)

Dexmedetomidine+morphine+midazolam 1 (5.0)

versus morphine+midazolam

Clonidine versus placebo

$1(5.0)$

Clonidine versus midazolam

$1(5.0)$

Clonidine+fentanyl +

$1(5.0)$

midazolam+thiopental versus

fentanyl+midazolam + thiopental

Clonidine+fentanyl + midazolam versus

$1(5.0)$

fentanyl+midazolam

Drug/protocol for sedation weaning

$3(10.3)$

Methadone+lorazepam (10 vs 5 days wean) 1 (33.3)

Methadone+diazepam (high vs low dose) 1 (33.3)

Methadone+lorazepam (protocolised regimen 1 (33.3)

vs standard care)

Daily interruption of sedation versus standard 3 (10.3) care

Morphine+midazolam

$1(33.3)$

Fentanyl+morphine
ketamine+propofol

Protocolised regimen versus standard care

3 (10.3)

Fentanyl+morphine +

1 (33.3)

remifentanil+hydromorphone versus

fentanyl+morphine + midazolam

Dexmedetomidine versus

midazolam+lorazepam + ketamine+propofol +

chloral hydrate +

\section{Barbiturates}

Morphine+midazolam + clonidine

$1(33.3)$

Intervention administrator (n, \%)

Physicians and nurses

$10(34.5)$

Physicians, nurses and other ancillary staff

3 (10.3)

Physicians alone

8 (27.6)

Nurses alone

$5(17.2)$

Not reported

$3(10.3)$

Continued
Table 3 Continued

Type of intervention (n, \%)

No of trials that reported ventilator management 10 (34.5) strategy $(n, \%)$

Secondary sedatives administered (n, \%)

Both control and comparator groups, same 17 (58.6) type of agents

Both control and comparator groups, but 7 (24.1)

different types of agents

No

$3(10.3)$

Not reported

$2(6.9)$

Neuromuscular blockade administered (n, \%)

Both control and comparator groups

No

$11(37.9)$

Not reported

$14(48.3)$

Duration of follow-up* (n, \%)

Until extubation

$5(17.2)$

24-96 hours after extubation

$3(10.3)$

Until sedatives discontinued

2 (6.9)

24-96 hours after sedatives discontinued

5 (17.2)

$>96$ hours after sedatives discontinued or until 2 (6.9)

no further decrease in sedatives were planned

Until treatment failure 1 (3.4)

Until discharged from hospital or PICU or $\quad 7$ (24.1)

transferred to another hospital

24-96 hours postoperative or not otherwise $3(10.3)$

specified

Up to 1 month, not otherwise specified 5 (17.2)

Up to 1 year, not otherwise specified 3 (10.3)

Not reported

$1(3.4)$

A summary of trial intervention, administration and follow-up duration is reported.

*Trials had various criteria for follow-up duration, which account for total number of trials exceeding $n=29$.

PICU, paediatric intensive care unit.

comparator $(27 / 29,93.1 \% ; 27 / 29,93.1 \%$, respectively $)$ groups were usually directed by an assigned protocol.

Twenty one $(72.4 \%)$ trials reported a single primary outcome, which included duration of mechanical ventilation $(8 / 29,27.6 \%)$, cumulative dose of sedative $(6 / 29$, $20.7 \%)$, time under adequate sedation $(4 / 29,13.8 \%)$ and rescue drug needed $(3 / 29,10.3 \%)$. In four trials $(13.8 \%)$, there were two or more primary outcome measures. In four trials (13.8\%), no primary outcome measure was defined.

\section{DISCUSSION}

This scoping review found that clinical trials evaluating sedation in critically ill paediatric patients differ considerably in their methodological approaches including general design and eligibility criteria, intervention types, 
Table 4 Efficacy assessment and outcome measures

Sedation assessment method* (n, \%)

\begin{tabular}{|c|c|}
\hline COMFORT scale & $7(24.1)$ \\
\hline COMFORT-behaviour scale & $6(20.7)$ \\
\hline Ramsay/modified ramsay sedation scale & $7(24.1)$ \\
\hline Richmond-agitation sedation scale & $1(3.4)$ \\
\hline State Behavioural Scale & $4(13.8)$ \\
\hline Nurse interpretation of sedation score & $2(6.9)$ \\
\hline Hartwig scale & $1(3.4)$ \\
\hline PICU sedation scale & $4(13.8)$ \\
\hline Modified motor activity assessment scale & $2(6.9)$ \\
\hline Tracheal suctioning score & $4(13.8)$ \\
\hline Riker Sedation-Agitation Scale & $1(3.4)$ \\
\hline Modified Narcotic Withdrawal Scale & $1(3.4)$ \\
\hline Other & $3(10.3)$ \\
\hline Not reported & $1(3.4)$ \\
\hline \multicolumn{2}{|l|}{ Pain assessment method* $(n, \%)$} \\
\hline $\begin{array}{l}\text { Faces, legs, activity, cry, consolability (or } \\
\text { modified) score }\end{array}$ & $6(20.7)$ \\
\hline Numeric Rating Scale & $3(10.3)$ \\
\hline Wong-Baker Faces Pain Scale & $2(6.9)$ \\
\hline Behavioural Pain Score & $1(3.4)$ \\
\hline Visual Analogue Score & $3(10.3)$ \\
\hline Not reported & $18(62.1)$ \\
\hline \multicolumn{2}{|l|}{ Sedation level assessor* (n, \%) } \\
\hline Physician (second party reporting) & $4(13.8)$ \\
\hline Researcher (second party reporting) & $6(20.7)$ \\
\hline Nurse (second party reporting) & $18(62.1)$ \\
\hline Not reported & $5(17.2)$ \\
\hline \multicolumn{2}{|l|}{ Frequency of sedation level assessment $(n, \%)$} \\
\hline Every 1 hour or more often & $7(24.1)$ \\
\hline Every $2-4$ hours & $19(65.5)$ \\
\hline Less frequent than every $2-4$ hours & $1(3.4)$ \\
\hline As clinically indicated, not otherwise specified & $2(6.9)$ \\
\hline
\end{tabular}

Sedation assessment in control group (n, \%)

$\begin{array}{lc}\text { Protocolised } & 25(86.2) \\ \text { Standard care } & 4(13.8) \\ \text { Sedation management in control group (n, \%) } & \\ \text { Protocolised } & 22(75.9) \\ \text { Individual physician judgement } & 2(6.9) \\ \text { Standard care } & 5(17.2)\end{array}$

Sedation assessment in comparator group (n,

\%)

$\begin{array}{lc}\text { Protocolised } & 27(93.1) \\ \text { Standard care } & 2(6.9)\end{array}$

Sedation management in comparator group (n, \%)

Protocolised

$27(93.1)$

Continued
Table 4 Continued

Sedation assessment method* (n, \%)

Standard care

$2(6.9)$

No of primary outcomes $(n, \%)$

1

$21(72.4)$

$\geq 2$

4 (13.8)

No outcome was identified as primary

$4(13.8)$

Primary outcome measure* $(n, \%)$

Cumulative dose of sedative

$6(20.7)$

Time under adequate sedation

4 (13.8)

Adequate sedation

2 (6.9)

Duration of mechanical ventilation (ie, time to 8 (27.6) extubation)

Length of PICU stay

$1(3.4)$

Rescue drug needed

3 (10.3)

Not reported

4 (13.8)

Other

$6(20.7)$

Completion of assigned taper

$1(3.4)$

Feasibility

$1(3.4)$

Ventilatory effects

$2(6.9)$

Biochemical markers

$1(3.4)$

Withdrawal syndrome

$1(3.4)$

Detailed assessment methods and outcome measures are described.

*Trials reported multiple sedation and pain assessment methods, assessors and primary outcome measures, which account for total number of trials exceeding $n=29$.

PICU, paediatric intensive care unit.

follow-up, assessments and outcome measures. Varying patient populations (eg, medical vs surgical, cardiac vs noncardiac, prolonged intubation vs 'fast track' or early extubation), polypharmaceutical approaches, and outcomes were investigated, which were further complicated by differences in the implementation of and adherence to protocols for sedation assessment and management. Such heterogeneity makes it difficult to compare results across studies and prevents the use of meta-analysis to synthesise evidence and establish generalisable practice recommendations. A number of gaps and inconsistencies in current trial design and reporting characteristics were identified in this review and warrant further discussion.

\section{Trial design}

Publications of trials were from various continents with the majority of publications occurring in this past decade. Most trials were small, single centre, and performed in academic children's hospitals. A substantial number of trials were not blinded in any fashion. While the absence of blinding may introduce a considerable source of bias, this finding should also be considered in the context of clinical constraints built-in to intensive care. Blinding was frequently described to be challenging or not feasible 
to execute depending on the nature of the trial and the paramount considerations of patient safety and clinical management.

No clear description of the rationale for sample size was reported in four trials. While this may have been a result of reporting omission rather than a deficiency in design, it is important to note given the fundamental nature of power calculation in quality trial design. A total $65.5 \%(19 / 29)$ of trials was able to enrol at least $90 \%$ of the planned sample size with a similar proportion of patients $(21 / 29,72.4 \%)$ who were able to complete the trial once enrolled. This is a positive finding for recruitment and retention of the trial subjects, since recruitment has historically been described as a major barrier to conducting paediatric trials. ${ }^{52}$ Challenges to recruitment were multifactorial due to the stress and complexity of the PICU environment. Consistently cited reasons included parental and provider reluctance, clinical work burden, as well as sensitivity in timing of consent. These difficulties may also reflect the greater issue of establishing consensus on equipoise in intensive care research, owing to significant variations in clinical practice as a result of patient-related, provider-related and site-related factors. Finally, trial completion was challenging, as several trials noted significant drop-out rates as the clinical course of enrolled subjects evolved over time. In our analysis, $67.9 \%$ $(19 / 28)$ of trials provided the CONSORT flow diagram, which made it difficult to assess trial characteristics.

\section{Eligibility criteria}

Based on the inclusion criteria, age spanned a wide range, but the enrolled patients were young with a median age of approximately 1.7 years. The vast majority of trials evaluated those patients who were mechanically ventilated, some of whom were expected to be extubated early and others requiring prolonged intubation. The time frame was not specified in 11 trials, and the lack of such specificity may be problematic since patients likely require different approaches for sedation based on the timing of extubation. Thus, outcomes may be expected to be quite variable for patients who had a relatively brief vs prolonged duration of mechanical ventilation.

Postsurgical patients, either with underlying cardiac or non-cardiac conditions, were the most commonly studied. These subpopulations should ideally be studied separately as unique approaches for sedation, analgesia and mechanical ventilation are often necessary. Common criteria for exclusion included the presence of neurological disease or other conditions impeding the evaluation of sedation level and the use of neuromuscular blockers. Severely ill, complex patients such as those with haemodynamic instability, major end-organ dysfunction, limited life expectancy or complex medical/surgical histories were also frequently excluded; these subsets of patients remain an understudied cohort. Given that paralytics are frequently administered in actual clinical practice, their exclusion from clinical trials may be problematic and limit generalisability. It was frequently noted that while stringent inclusion and exclusion criteria may improve homogeneity of the trial population, recruitment and retention, in turn, become more challenging.

\section{Intervention characteristics}

This review found intervention type varied and mostly compared unique drugs and/or protocols for sedation. Sedation weaning, interruption and protocolisation were also areas of interest. Many trials reported the administration of secondary sedatives in addition to those specific drugs being evaluated, with approximately a quarter of trials describing a divergent profile of drugs between the control and comparator arms. While the administration of multiple sedatives and analgesics is expected in the critical care setting where the escalation of care is commonplace, this serious confounding makes it difficult to meaningfully interpret the data. Other areas that were not well described in the majority of trials, such as ventilator management strategy and use of neuromuscular blockade, may be additional confounding factors.

Adherence to protocol is a critical element in trials, but this was only assessed in $31.0 \%(9 / 29)$ of trials. Similarly, the use of pretrial training and a run-in period was generally rare. While these processes would be ideal to ensure adequate quality, their labour-intensive, time-intensive and cost-intensive nature may limit their execution in real practice. Finally, the duration of follow-up was heterogeneous in clinical trials, but typically short term, on the order of days.

\section{Efficacy assessment and outcome measures}

Sedation assessment was found to be regularly protocolised with frequent assessments performed most often by bedside nurses. Management of sedation was also directed by an established protocol in the vast majority of cases, though protocol adherence was not usually evaluated as discussed previously. Instruments used to assess sedation levels varied considerably. The COMFORT or COMFORTBehaviour Scales were the most frequently used, though this varied depending on the age of enrolled subjects and geography. Although these instruments have been extensively studied and validated for the assessment of sedation and analgesia in PICU patients, ${ }^{57}$ a significant limiting factor is that they cannot accurately score sedation or pain when patients are paralysed. ${ }^{57} 58$ The second most frequently used instrument was the Ramsay/Modified Ramsay Sedation Scale, but this instrument has only been validated for adult ICU patients. ${ }^{57}$ Additionally, while these scales can adequately assess sedation, they have not been designed to evaluate pain. ${ }^{5758}$

We found that the scoring of pain, distinct from sedation level, was not reported in the majority of trials. The discrimination of inadequate sedation versus analgesia is inherently a difficult clinical problem, though may be important to consider carefully when evaluating optimal instrument selection. This issue is particularly challenging when caring for paediatric patients who are often unable to verbalise their symptoms or will fully cooperate 
with necessary interventions and bedside care. Indeed, oversedation has been reported to be more of an issue compared with undersedation in the PICU setting. ${ }^{16}$ This is likely due to a myriad of issues including difficulties with identifying a patient's needs, particularly in those aforementioned younger, preverbal patients and a desire to avoid the serious risks of undersedation at all costs such as self-extubation or the removal of lines and devices. Weaning and optimising sedation levels may also naturally be overlooked or suspended when urgent clinical matters requiring immediate attention arise.

The most common primary outcome measures included duration of mechanical ventilation, cumulative dose of sedation, time under adequate sedation and rescue drug needed. While these may be appropriate surrogates to assess clinical efficacy, there are certainly other important outcome dimensions in sedation management that merit consideration for further study. Patientcentred and/or family-centred outcomes is one such example of an outcome domain that is understudied, but may be important to consider, particularly in the paediatric population. ${ }^{59}$ The level of patient and/or family satisfaction with the sedation experience can provide a valuable assessment of the effects of analgesia, anxiolysis, amnesia, as well as absence or presence of unfavourable outcomes, all of which may have a significant impact on sedation management (eg, patient and/or family compliance, provider decision making).$^{20}$ In addition, while it is recognised that there needs to be a better understanding of the effects of sedation on longer-term outcomes, such as cognition or other brain health-related outcomes, currently, there remains a need for further research. ${ }^{60-62}$ Outcomes selection should be balanced based on clinical relevance, scientific validity, and feasibility to optimise trial success.

Considerations for future trial design and conduct are proposed in this review (table 5), but these may not be suitable for all sedation trials. An adaptation of these considerations may be more appropriate to be applied to the unique aspects of individual trial objectives and design. The pragmatic trial design may be a useful option to consider as its unique approach allows for broad patient recruitment and the evaluation of interventions in real life, complex practice conditions, which are subject to the influences of various forces present in an open system like the PICU. ${ }^{63-65}$ Pragmatic trials may also be particularly valuable in scenarios where blinding is infeasible, a limitation which was discussed earlier. Cluster design trials with or without stepped-wedge elements, which involve random and sequential crossover of clusters from control to intervention until all clusters are exposed, are another example of a suitable design approach. ${ }^{31}$ The Sedation AND Weaning in Children trial ${ }^{54}$ provides an exemplary model for how a pragmatic multicentre, stepped-wedge, cluster trial may be successfully carried out in the PICU setting.

Table 5 Design and reporting considerations for paediatric sedation clinical trials in the ICU

\begin{tabular}{|c|c|}
\hline Category & Considerations \\
\hline Eligibility & $\begin{array}{l}\text { Report inclusion and exclusion criteria. } \\
\text { Consider how clinical criteria for trial participants (eg, medical vs surgical, cardiac vs non-cardiac) may } \\
\text { affect outcome analysis. } \\
\text { If mechanical ventilation is an inclusion criterion, report ventilation strategy and specific parameters in } \\
\text { terms of expected duration of ventilation and its plausible effect on outcome analysis. }\end{array}$ \\
\hline $\begin{array}{l}\text { Recruitment } \\
\text { and retention }\end{array}$ & $\begin{array}{l}\text { Consider broader inclusion criteria to facilitate recruitment and retention. } \\
\text { Consider collaboration across multiple sites to ensure adequate statistical power. } \\
\text { Report sample size calculation and rationale. } \\
\text { Report CONSORT flow diagram to describe all trial phases (screening, enrolment, randomisation and } \\
\text { completion). } \\
\text { Discuss any challenges with recruitment, retention and adherence and any contributors to either success or } \\
\text { failure. }\end{array}$ \\
\hline $\begin{array}{l}\text { Intervention, } \\
\text { adherence and } \\
\text { blinding }\end{array}$ & $\begin{array}{l}\text { Describe intended intervention and trial protocols. } \\
\text { Consider monitoring protocol violations and assessing adherence to defined intervention. } \\
\text { Consider the use of pretrial training or a run-in period to promote adherence } \\
\text { Report the presence or absence of blinding to the intervention and any challenges therein. } \\
\text { Monitor and encourage the use of a consistent set of adjuvant sedatives and paralytics in control and } \\
\text { comparator arms. }\end{array}$ \\
\hline $\begin{array}{l}\text { Efficacy } \\
\text { assessment } \\
\text { and outcome } \\
\text { measures }\end{array}$ & $\begin{array}{l}\text { Report the primary outcome measure and whether it was prespecified. } \\
\text { Indicate how and by whom the outcome measure was assessed. } \\
\text { Consider strengths and limitations of assessment instruments during selection process. } \\
\text { Consider extending follow-up duration to include longer term outcomes when possible. } \\
\text { Obtain stakeholder input and consensus to develop a core set of efficacy outcomes. }\end{array}$ \\
\hline
\end{tabular}

Considerations for future trial design and reporting based on this review's results are summarised.

CONSORT, Consolidated Standards of Reporting Trials; ICUs, intensive care units. 


\section{Strengths and limitations}

Strengths of this review include strict adherence to PRISMA guidelines and an exhaustive search strategy to identify all relevant trials up to date, including a snowballing strategy and looking at grey literature. Data extraction of an extensive range of design and reporting elements was executed, and key issues in trial methodology were uncovered.

This review is limited in that we mainly looked at publications of paediatric sedation trials in peer-reviewed literature. Prospective observational and other interventional studies such as before-and-after studies were excluded given the aim of this review was to review trial design and reporting. We acknowledge the significant prevalence and value of other types of studies in informing the paediatric critical care literature addressing sedation practices, and a separate review describing these studies may be worth conducting. We view this review's findings as a starting point for stakeholders' discussions (eg, multidisciplinary experts from academia, industry, FDA, patients and their families) and acknowledge the numerous challenges inherent to the PICU setting, which make the execution of clinical trials exquisitely complex. Lastly, safety and adverse events reporting was not discussed within this review given its present focus on general design and reporting of efficacy outcomes.

\section{CONCLUSIONS}

This scoping review examined paediatric sedation clinical trials in the critically ill population and found considerable heterogeneity in design and reporting characteristics. Current gaps and variations in eligibility criteria, intervention characteristics and efficacy outcome measures were summarised, and considerations for trial design and execution were proposed. Findings from this review may provide a basis for the development of a core set of trial design recommendations and outcome domains for sedation trials in paediatric critical care patients, which may improve the quality and comparability of future clinical trials. Input and consensus from various stakeholders is needed to establish evidence-based practice guidelines.

\section{Author affiliations}

${ }^{1}$ Anesthesiology, Columbia University Irving Medical Center, New York, New York, USA

${ }^{2}$ Anesthesiology and Perioperative Medicine, University of Rochester Medical Center, Rochester, New York, USA

${ }^{3}$ Pediatrics, Stony Brook University Renaissance School of Medicine, Stony Brook, New York, USA

${ }^{4}$ Anesthesiology, Critical Care and Pediatrics, University of Pennsylvania Perelman School of Medicine, Philadelphia, Pennsylvania, USA

${ }^{5}$ Anesthesiology and Pediatrics, Columbia University Irving Medical Center, New York, New York, USA

Acknowledgements This article was supported by SCEPTER established by the ACTTION public-private partnership with the US FDA. The authors thank John Usseglio, M.P.H., of the Columbia University Irving Medical Center Health Sciences Library, New York, New York, USA for his assistance in conducting the literature searches during the conception of this work.
Contributors Conception and design of the review: LSS, RHD, JJL and JCP. Article screening and data extraction: JJL and JCP. Contribution to development of methodology: JG and BAK. Data analysis and interpretation: JJL, JCP, JG, BAK, KVB, MYN, DW, RHD and LSS. JJL drafted, and all authors critically reviewed the manuscript. All authors read and approved the manuscript.

Funding The authors have not declared a specific grant for this research from any funding agency in the public, commercial or not-for-profit sectors.

Competing interests None declared.

Patient consent for publication Not applicable.

Ethics approval This study does not involve human participants or animal subjects.

Provenance and peer review Not commissioned; externally peer reviewed.

Data availability statement All data relevant to the study are included in the article or uploaded as online supplemental information.

Supplemental material This content has been supplied by the author(s). It has not been vetted by BMJ Publishing Group Limited (BMJ) and may not have been peer-reviewed. Any opinions or recommendations discussed are solely those of the author(s) and are not endorsed by BMJ. BMJ disclaims all liability and responsibility arising from any reliance placed on the content. Where the content includes any translated material, BMJ does not warrant the accuracy and reliability of the translations (including but not limited to local regulations, clinical guidelines, terminology, drug names and drug dosages), and is not responsible for any error and/or omissions arising from translation and adaptation or otherwise.

Open access This is an open access article distributed in accordance with the Creative Commons Attribution Non Commercial (CC BY-NC 4.0) license, which permits others to distribute, remix, adapt, build upon this work non-commercially, and license their derivative works on different terms, provided the original work is properly cited, appropriate credit is given, any changes made indicated, and the use is non-commercial. See: http://creativecommons.org/licenses/by-nc/4.0/.

\section{ORCID iDs}

Jennifer Jooyoung Lee http://orcid.org/0000-0001-5456-1688

Maryam Y Naim http://orcid.org/0000-0002-9127-0043

\section{REFERENCES}

1 Vet NJ, Kleiber N, Ista E, et al. Sedation in critically ill children with respiratory failure. Front Pediatr 2016;4:1-15.

2 Jacobi J, Fraser GL, Coursin DB, et al. Clinical practice guidelines for the sustained use of sedatives and analgesics in the critically ill adult. Crit Care Med 2002;30:119-41.

3 Harris J, Ramelet A-S, van Dijk M, et al. Clinical recommendations for pain, sedation, withdrawal and delirium assessment in critically ill infants and children: an ESPNIC position statement for healthcare professionals. Intensive Care Med 2016;42:972-86.

4 Playfor S, Jenkins I, Boyles C, et al. Consensus guidelines on sedation and analgesia in critically ill children. Intensive Care Med 2006;32:1125-36.

5 Lucas SS, Nasr VG, Ng AJ, et al. Pediatric cardiac intensive care Society 2014 consensus statement: pharmacotherapies in cardiac critical care: sedation, analgesia and muscle relaxant. Pediatr Crit Care Med 2016;17:S3-15.

6 Dellinger RP, Levy MM, Rhodes A, et al. Surviving sepsis campaign: international guidelines for management of severe sepsis and septic shock: 2012. Crit Care Med 2013;41:580-637.

7 Kochanek PM, Carney N, Adelson PD, et al. Guidelines for the acute medical management of severe traumatic brain injury in infants, children, and adolescents--second edition. Pediatr Crit Care Med 2012;13:S1.

8 Brierley J, Carcillo JA, Choong K, et al. Clinical practice parameters for hemodynamic support of pediatric and neonatal septic shock: 2007 update from the American College of critical care medicine. Crit Care Med 2009;37:666-88.

9 Twite MD, Rashid A, Zuk J, et al. Sedation, analgesia, and neuromuscular blockade in the pediatric intensive care unit: survey of fellowship training programs. Pediatr Crit Care Med 2004;5:521-32.

10 Best KM, Asaro LA, Franck LS, et al. Patterns of sedation weaning in critically ill children recovering from acute respiratory failure. Pediatr Crit Care Med 2016;17:19-29.

11 Rhoney DH, Murry KR. National survey on the use of sedatives and neuromuscular blocking agents in the pediatric intensive care unit. Pediatr Crit Care Med 2002;3:129-33. 
12 Doherty DR, Pascuet E, Ni A, et al. Off-label drug use in pediatric anesthesia and intensive care according to official and pediatric reference formularies. Can J Anaesth 2010;57:1078-88.

13 Carvalho PRA, Carvalho CG, Alievi PT, et al. [Prescription of drugs not appropriate for children in a pediatric intensive care unit. $J$ Pediatr 2003;79:397-402.

14 Bavdekar SB, Sadawarte PA, Gogtay NJ, et al. Off-label drug use in a pediatric intensive care unit. Indian J Pediatr 2009;76:1113-8.

15 Yang CP, Veltri MA, Anton B, et al. Food and drug administration approval for medications used in the pediatric intensive care unit: a continuing conundrum. Pediatr Crit Care Med 2011;12:e195-9.

16 Vet NJ, Ista E, de Wildt SN, et al. Optimal sedation in pediatric intensive care patients: a systematic review. Intensive Care Med 2013;39:1524-34.

17 Duffett M, Choong K, Hartling L, et al. Randomized controlled trials in pediatric critical care: a scoping review. Crit Care 2013;17:R256.

18 Williams MR, McKeown A, Dexter F, et al. Efficacy outcome measures for procedural sedation clinical trials in adults: an ACTTION systematic review. Anesth Analg 2016;122:152-70.

19 Williams MR, Nayshtut M, Hoefnagel A, et al. Efficacy outcome measures for pediatric procedural sedation clinical trials: an ACTTION systematic review. Anesth Analg 2018;126:956-67.

20 Williams MR, Ward DS, Carlson D, et al. Evaluating patientcentered outcomes in clinical trials of procedural sedation, part 1 efficacy: sedation Consortium on endpoints and procedures for treatment, education, and research recommendations. Anesth Analg 2017;124:821-30.

21 Ward DS, Williams MR, Berkenbosch JW, et al. Evaluating patientcentered outcomes in clinical trials of procedural sedation, part 2 safety: sedation Consortium on endpoints and procedures for treatment, education, and research recommendations. Anesth Analg 2018;127:1146-54

22 Ward DS, Absalom A, Aitken LM. Design of clinical trials evaluating sedation in critically ill adults undergoing mechanical ventilation: recommendations from sedation Consortium on endpoints and procedures for treatment, education, and research (SCEPTER) recommendations III. Crit Care Med2021.

23 Tricco AC, Lillie E, Zarin W, et al. PRISMA extension for scoping reviews (PRISMA-ScR): checklist and explanation. Ann Intern Med 2018;169:467-73.

24 Covidence systematic review software, veritas health innovation, Melbourne, Australia. Available: www.covidence.org

25 Duffet M. Randomized controlled trials in pediatric intensive care. Available: picutrials.net [Accessed $10 \mathrm{Dec} 2020]$

26 Akinci SB, Kanbak M, Guler A, et al. Remifentanil versus fentanyl for short-term analgesia-based sedation in mechanically ventilated postoperative children. Paediatr Anaesth 2005;15:870-8.

27 Aydogan MS, Korkmaz MF, Ozgül U, et al. Pain, fentanyl consumption, and delirium in adolescents after scoliosis surgery: dexmedetomidine vs midazolam. Paediatr Anaesth 2013;23:446-52.

28 Berens RJ, Meyer MT, Mikhailov TA, et al. A prospective evaluation of opioid weaning in opioid-dependent pediatric critical care patients. Anesth Analg 2006;102:1045-50.

29 Bouwmeester NJ, Anand KJ, van Dijk M, et al. Hormonal and metabolic stress responses after major surgery in children aged 0-3 years: a double-blind, randomized trial comparing the effects of continuous versus intermittent morphine. Br J Anaesth 2001:87:390-9.

30 Bowens CD, Thompson JA, Thompson MT, et al. A trial of methadone tapering schedules in pediatric intensive care unit patients exposed to prolonged sedative infusions. Pediatr Crit Care Med 2011;12:504-11.

31 Curley MAQ, Wypij D, Watson RS, et al. Protocolized sedation vs usual care in pediatric patients mechanically ventilated for acute respiratory failure: a randomized clinical trial. JAMA 2015;313:379-89.

32 Darnell CM, Thompson J, Stromberg D, et al. Effect of low-dose naloxone infusion on fentanyl requirements in critically ill children. Pediatrics 2008:121:e1363-71.

33 da Silva PSL, Reis ME, de Aguiar VE, et al. Use of fentanyl and midazolam in mechanically ventilated children--Does the method of infusion matter? J Crit Care 2016;32:108-13.

34 Duffett $\mathrm{M}$, Choong K, Foster J, et al. Clonidine in the sedation of mechanically ventilated children: a pilot randomized trial. J Crit Care 2014;29:758-63.

35 Erickson SJ, Millar J, Anderson BJ, et al. Dexmedetomidine sedation in mechanically ventilated critically ill children: a pilot randomized controlled trial. Pediatr Crit Care Med 2020;21:e731-9.

36 Garisto C, Ricci Z, Tofani L, et al. Use of low-dose dexmedetomidine in combination with opioids and midazolam in pediatric cardiac surgical patients: randomized controlled trial. Minerva Anestesiol 2018:84:1053-62.

37 Gupta K, Gupta VK, Jayashree M, et al. Randomized controlled trial of interrupted versus continuous sedative infusions in ventilated children. Pediatr Crit Care Med 2012;13:131-5.

38 Hünseler C, Balling G, Röhlig C, et al. Continuous infusion of clonidine in ventilated newborns and infants: a randomized controlled trial. Pediatr Crit Care Med 2014;15:511-22.

39 Lee B, Park JD, Choi YH, et al. Efficacy and safety of fentanyl in combination with midazolam in children on mechanical ventilation. $J$ Korean Med Sci 2019;34:e21

40 Macnab AJ, Levine M, Glick N, et al. Midazolam following open heart surgery in children: haemodynamic effects of a loading dose. Paediatr Anaesth 1996;6:387-97.

41 Parkinson L, Hughes J, Gill A, et al. A randomized controlled trial of sedation in the critically ill. Paediatr Anaesth 1997;7:405-510.

42 Penk JS, Lefaiver CA, Brady CM, et al. Intermittent versus continuous and intermittent medications for pain and sedation after pediatric cardiothoracic surgery; a randomized controlled trial. Crit Care Med 2018:46:123-9.

43 Prasad SR, Simha PP, Jagadeesh AM. Comparative study between dexmedetomidine and fentanyl for sedation during mechanical ventilation in post-operative paediatric cardiac surgical patients. Indian J Anaesth 2012;56:547-52.

44 Salarian S, Khosravi R, Khanbabaei G, et al. Impact of oral clonidine on duration of opioid and benzodiazepine use in mechanically ventilated children: a randomized, double-blind, placebo-controlled study. Iran J Pharm Res 2019;18:2157-62.

45 Hussein R. Randomized prospective double-blinded comparative study between dexmedetomidine and fentanyl for sedation in children post-operative paediatric surgical intensive care unit. AAMJ 2013;11:334-47.

46 Saleh RH. Randomized controlled comparative trial between low dose dexmedetomidine sedation and that of fentanyl in children after surgical procedures in surgical pediatric intensive care unit. Egypt $J$ Anaesth 2016;32:137-42.

47 Tiacharoen D, Lertbunrian R, Veawpanich J, et al. Protocolized sedative weaning vs usual care in pediatric critically ill patients: a pilot randomized controlled trial. Indian J Crit Care Med 2020;24:451-8

48 Tobias JD, Berkenbosch JW. Sedation during mechanical ventilation in infants and children: dexmedetomidine versus midazolam. South Med J 2004;97:451-5.

49 van Dijk M, Bouwmeester NJ, Duivenvoorden HJ, et al. Efficacy of continuous versus intermittent morphine administration after major surgery in 0-3-year-old infants; a double-blind randomized controlled trial. Pain 2002;98:305-13.

50 Verlaat CWM, Heesen GP, Vet NJ, et al. Randomized controlled trial of daily interruption of sedatives in critically ill children. Paediatr Anaesth 2014;24:151-6.

51 Vet NJ, de Wildt SN, Verlaat CWM, et al. A randomized controlled trial of daily sedation interruption in critically ill children. Intensive Care Med 2016;42:233-44.

52 Wolf A, McKay A, Spowart C, et al. Prospective multicentre randomised, double-blind, equivalence study comparing clonidine and midazolam as intravenous sedative agents in critically ill children the SLEEPS (safety profiLe, efficacy and equivalence in paediatric intensive care sedation) study. Health Technol Assess 2014;18:1-212.

53 Gulla KM, Sankar J, Jat KR, et al. Dexmedetomidine vs midazolam for sedation in mechanically ventilated children: a randomized controlled trial. Indian Pediatr 2021;58:117-22.

54 Blackwood B, Tume LN, Morris KP, et al. Effect of a sedation and ventilator liberation protocol vs usual care on duration of invasive mechanical ventilation in pediatric intensive care units: a randomized clinical trial. JAMA 2021;326:401-10.

55 Begg Cet al. Improving the quality of reporting of randomized controlled trials. JAMA 1996;276:637-9.

56 Center for drug evaluation and research world training: office of new drugs module, pediatric drug legislation. Available: https://www. accessdata.fda.gov/scripts/cderworld/index.cfm?action=newdrugs: main [Accessed 5 Apr 2021]

57 Vet NJ, Kleiber N, Ista E. Sedation in critically ill children with respiratory failure. Front Pediatr 2016;89:1-15.

58 Dorfman TL, Sumamo Schellenberg E, Rempel GR, et al. An evaluation of instruments for scoring physiological and behavioral cues of pain, non-pain related distress, and adequacy of analgesia and sedation in pediatric mechanically ventilated patients: a systematic review. Int J Nurs Stud 2014;51:654-76.

59 Leavitt M, Wolfe A. Medscape's response to the Institute of medicine report: crossing the quality chasm: a new health system for the 21 st century. MedGenMed 2001;3:233-5. 
60 Fink EL, Tasker RC. Brain-related outcome measures in trials recruiting critically-ill children. Curr Opin Pediatr 2019;31:775-82.

61 Curley MAQ, Watson RS, Cassidy AM, et al. Design and rationale of the "Sedation strategy and cognitive outcome after critical illness in early childhood" study. Contemp Clin Trials 2018;72:8-15.

62 Watson RS, Asaro LA, Hertzog JH, et al. Long-Term outcomes after Protocolized sedation versus usual care in ventilated pediatric patients. Am J Respir Crit Care Med 2018;197:1457-67.
63 Branch-Elliman W, Lehmann LS, Boden WE, et al. Pragmatic, adaptive clinical trials: is 2020 the dawning of a new age? Contemp Clin Trials Commun 2020;19:100614.

64 Dal-Ré R, Janiaud P, loannidis JPA. Real-world evidence: how pragmatic are randomized controlled trials labeled as pragmatic? BMC Med 2018;16:49.

65 Ford I, Norrie J. Pragmatic trials. N Engl J Med 2016;375:454-63. 